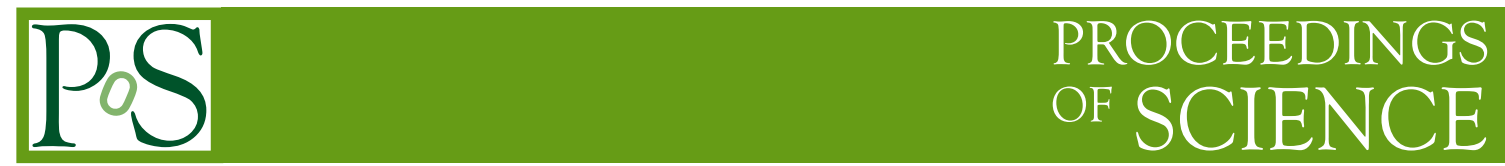

\title{
SW Sex Stars Then and Now: A Review
}

\author{
Linda Schmidtobreick* \\ European Southern Observatory, Casilla 19001, Santiago 19, Chile \\ E-mail: Ischmidteeso.org
}

SW Sextantis stars are a class of cataclysmic variables originally defined via certain peculiar properties that they all have in common. In this article, I review our knowledge of these stars and show the way from a phenomenological classification to a physical understanding of these systems. The fact that SW Sex stars accumulate at the upper edge of the period gap is discussed with respect to the secular evolution of cataclysmic variables.

The Golden Age of Cataclysmic Variables and Related Objects - III, Golden2015

7-12 September 2015

Palermo, Italy

${ }^{*}$ Speaker. 


\section{Some History}

About 25 years ago, the mysterious but consistent and reproducible behaviour of four stars was mentioned for the first time by Vik Dhillon [6] and Thorstensen et al. [39]. The stars, SW Sex, DW UMa, V1315 Aql and PX And were characterised as eclipsing novalike systems which however showed single-peaked emission lines (see e.g. Figure 1) instead of the double-peaked lines one would expect for high-inclination accretion discs [16]. The orbital periods of these systems lie in the range between 3 and 4 hours, i.e. the region above the period gap. They seemed to be nonmagnetic, as no polarisation or X-rays had been found. Nevertheless, HeÍI was noted as strong, about half the strength of $\mathrm{H} \beta$ which is usually a sign for magnetic CVs. Thorstensen et al. point out that "the radial velocities of the emission lines vary periodically, but the Balmer line velocities lag substantially behind the phase one expects for the white dwarf on the basis of the eclipse."[39] (see Figure 1 for an example). The final defining feature are transient absorption lines that appear only during opposite eclipse phases; an example is shown in Figure 1. The group of stars that fullfill these constraints is referred to as the SW Sex stars (see Table 1 for a summary).

After the initial classification scheme was developed, several more systems were found that fell into the SW Sex category, e.g. BH Lyn [7], WX Ari [4] or V795 Her [5], and also two old novae BT Mon [37] and V533 Her [38]. At the same time, first attempts were made to understand the SW Sex phenomenon and the mechanism responsible for the mysterious features. Trailed spectra and Doppler maps were computed to understand the distribution of the emission line sources, see e.g. Figure 2 and 3.

In general, SW Sex stars were recognised as a rare sub-species of CVs showing peculiar be-
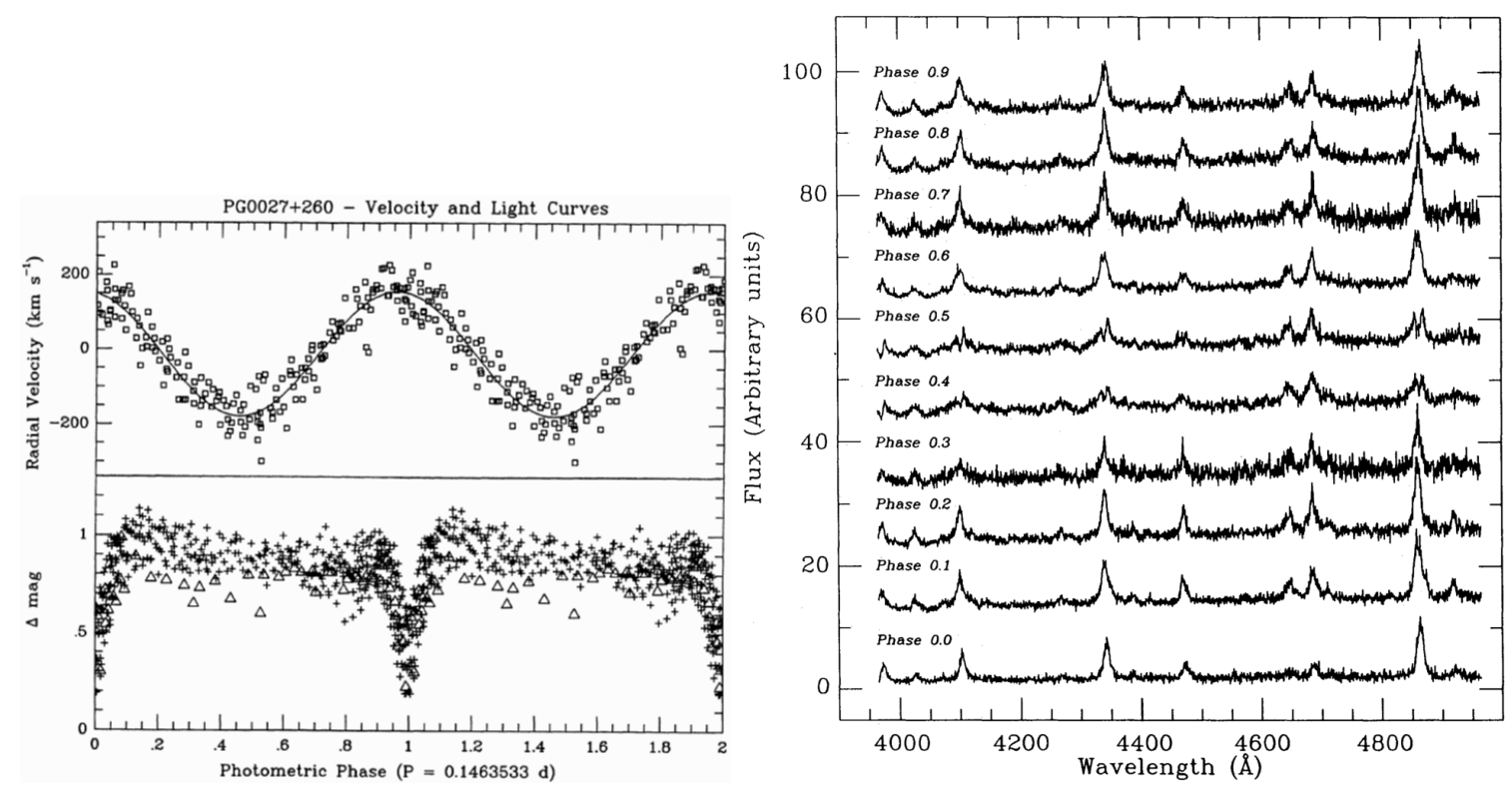

Figure 1: Some examples for the originally defining features of SW Se stars. On the left side, the radial velocities of $\mathrm{H} \alpha$ (top) and differential V magnitudes (bottom) of PX And are plotted against the orbital phase. The zero-crossing towards negative velocities, which should occur at phase 0 and 1 for the motion of the white dwarf, happens around phase 1.2; the velocities just lag behind the expected ones [39]). On the right side, the phase-resolved spectra of V1315 Aql show the absorption feature at phase 0.5 , the eclipse is at 0.0 [6]. 

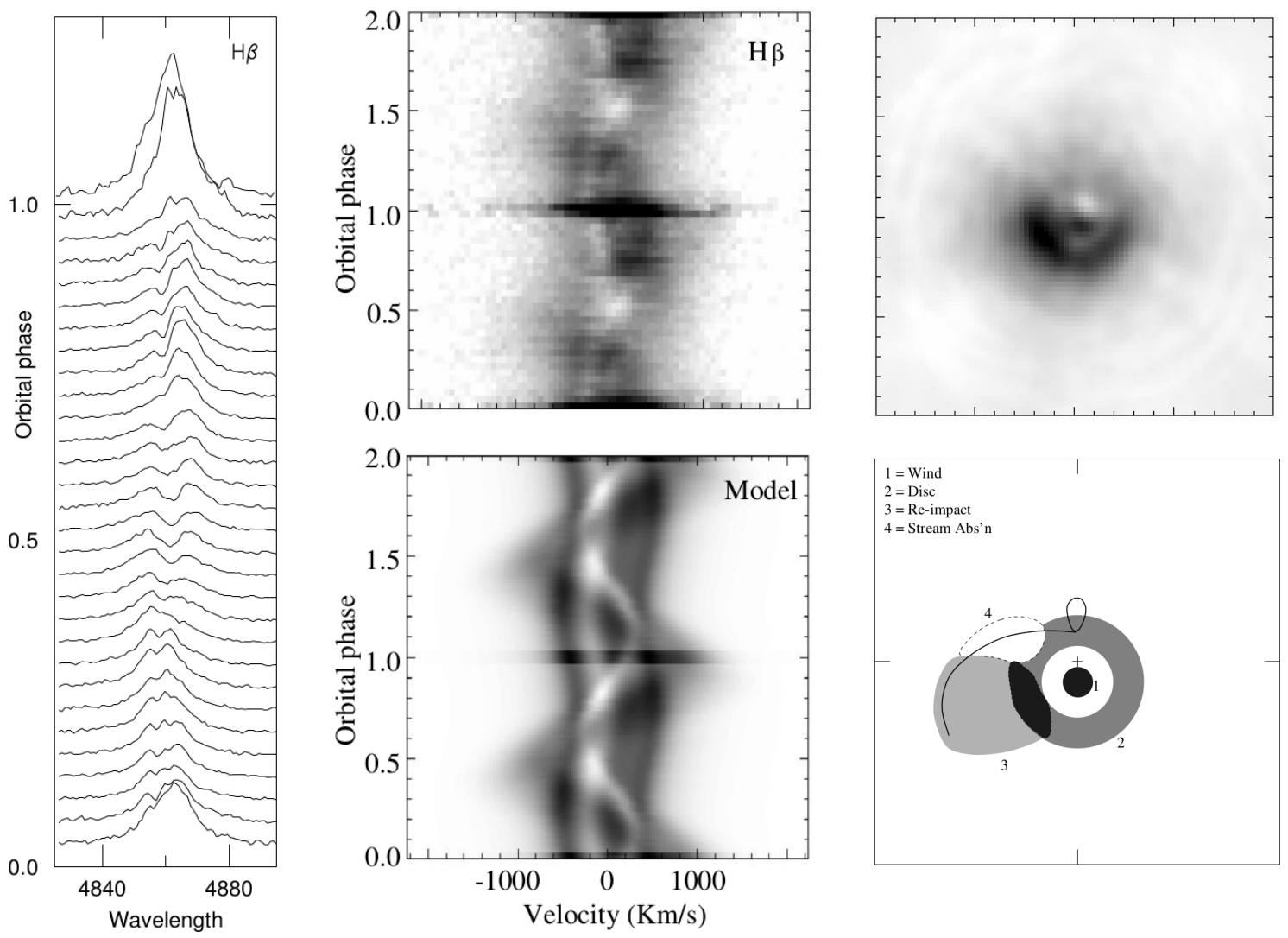

Figure 2: The orbitally phase-binned $\mathrm{H} \beta$ line profiles on the left show the absorption feature around phase 0.5 . In the centre, the trailed spectra of $\mathrm{H} \beta$ reveals the complex composition of the emission line including the high velocity wings. For the model below, emission from a wind, the disk, and the re-impact site, together with P Cygni absorption and stream absorption are taken into account. On the right side, the Doppler map of the $\mathrm{H} \beta$ emission is plotted, below a schematic interpretation of the tomogram is given showing the position of the various components that contribute to the emission line profile. All plots are taken from Hellier [11].
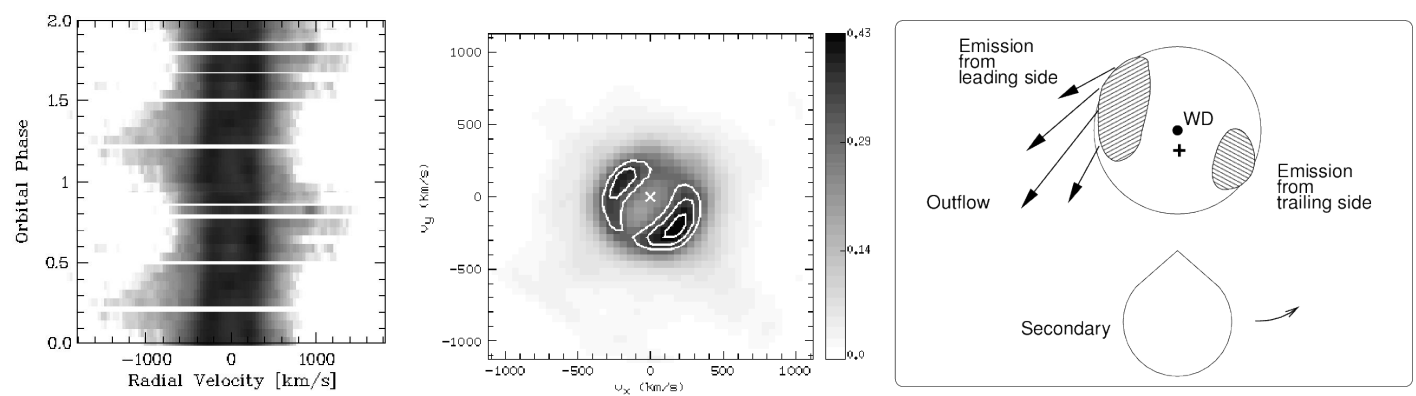

Figure 3: Trailed spectra of $\mathrm{H} \alpha$ (left) and the Doppler map of the $\mathrm{H} \alpha$ emission (centre) are plotted for RR Pic. On the right side, a schematic interpretation of the data is given. All plots are taken from Schmidtobreick et al. [36]). 
Table 1: The first definition of SW Sex stars as a new class of cataclysmic variables was done with four systems [39]. See text for details on the defining features.

\begin{tabular}{|l|c|c|c|c|c|c|}
\hline System & eclipsing & single peak & absorption & RV phase-lag & strong HeII & period/d \\
\hline \hline SW Sex & $\sqrt{ }$ & $\sqrt{ }$ & $\sqrt{ }$ & $\sqrt{ }$ & $\sqrt{ }$ & 0.134938 \\
DW UMa & $\sqrt{ }$ & $\sqrt{ }$ & $\sqrt{ }$ & $\sqrt{ }$ & $\sqrt{ }$ & 0.136607 \\
V1315 Aql & $\sqrt{ }$ & $\sqrt{ }$ & $\sqrt{ }$ & $\sqrt{ }$ & $\sqrt{ }$ & 0.139690 \\
PX And & $\sqrt{ }$ & $\sqrt{ }$ & $\sqrt{ }$ & $\sqrt{ }$ & $\sqrt{ }$ & 0.146353 \\
\hline
\end{tabular}

haviour. However, already Thorstensen et al. 1991 found the amount of discovered SW Sex systems remarkable, i.e. $3 / 30$ in the PG survey, especially as they have to be seen at high inclination to be discovered as such. They noted that 'the weird absorption events, the phase-displaced emission lines, and so on, must be regarded as normal rather than pathological' [39]. And indeed, with all the surveys coming up in the new century, the number of newly found SW Sex candidates exploded (see Figure 4 for a summary of the surveys). In particular, all eclipsing novalike stars with an orbital period between 3 and 4 hours, are of SW Sex nature (Gänsicke 2005 [10]). However, whether a binary is eclipsing or not, depends on its inclination but not on its physical properties. Thus, it is reasonable to assume that all non- or weakly-magnetic CVs with orbital periods between 3 and 4 hours share the physical properties of SW Sex stars. Follow-up observations of non-eclipsing CVs in this period range showed indeed that the large majority of these systems present at least some of the defining SW Sex features ([32], [33], [35]). Rather than some exotic sub-species of CVs, SW Sex stars turn out to be the dominant population in the 3-4 hour period range. At the writing of this paper, a total of $73 \mathrm{CVs}^{1}$ are considered to be possible SW Sex stars.

\section{The physics of the SW Sex features}

Already at the very beginning of the SW Sex studies, people were wondering about the physical explanation for the various SW Sex phenomena. The reason, SW Sex stars had been identified as a distinctive class, was that several of the observed characteristics contradicted the general understanding of how CVs were built-up. Honeycutt et al. [15] discussed the transient absorption as
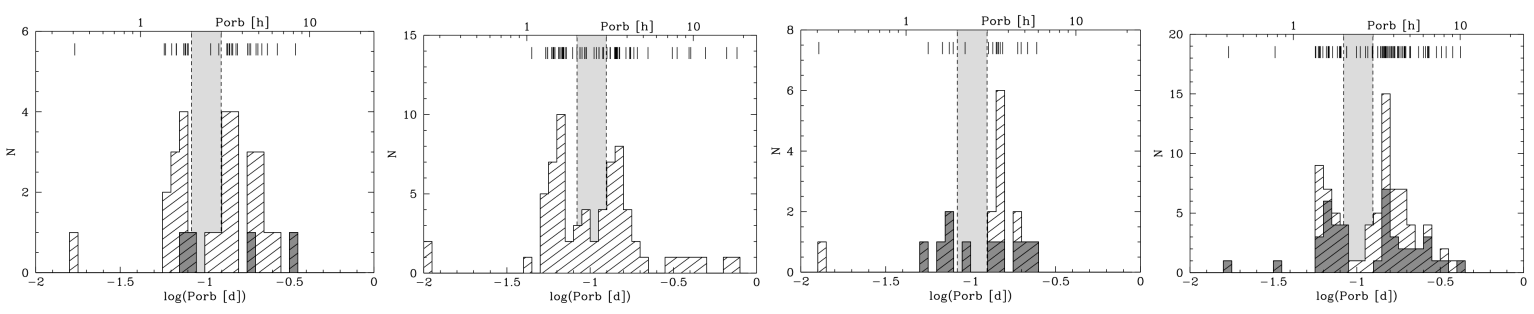

Figure 4: The orbital period distribution of CVs as found in various surveys from the beginning of the 21st century. From left to right: Palomar Green, ROSAT, Edinburgh Cape and Hamburg Quasar survey. In all plots, the period gap is indicated with a light grey bar, previously known systems are shown in dark grey. All surveys have discovered a large number of CVs with orbital periods between 3 and $4 \mathrm{~h}$ - the regime of the SW Sex stars (white bar left of the period gap). Taken from [10].

\footnotetext{
${ }^{1}$ See D. W. Hoard's Big List of SW Sextantis Stars at www. dwhoard.com/biglist [14]
} 


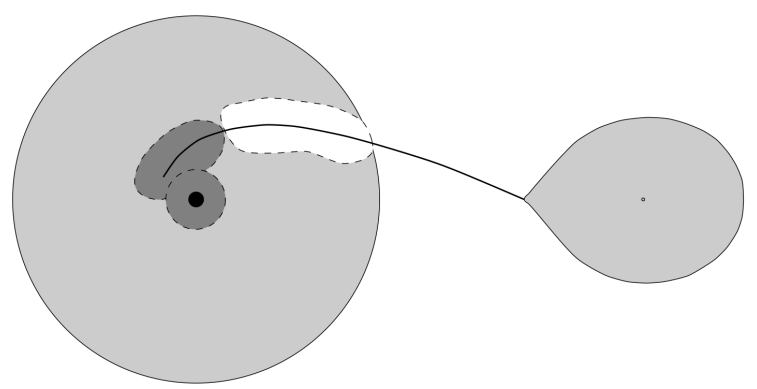

Figure 5: A schematic illustration of Hellier's model for SW Sex stars with a disk overflow causing absorption (white region) and then emission (dark region). An accretion disk wind from near the white dwarf adds to the emission, filling in the double-peaked profile of the accretion disk. [11]

an effect due to a somewhat discontinuous s-wave or to the appearance of an absorption component near inferior conjunction. To explain both, the transient absorption and the single-line profiles in an eclipsing binary, Hellier [11] developed a model of a disc overflow together with an accretion wind that would fill in the double-peaked line profile from the accretion disc (see Figure 5). He suggested that such an overflow and wind could be the result of a high mass-transfer rate.

There are indeed more indications that SW Sex stars are CVs that experience extremely high mass transfer rates. Already Thorstensen et al. noted in 1991 that 'the characteristics of the SW Sex stars suggest that, except for occasional low states, they are persistently bright novalike variables. All were discovered in magnitude-limited searches, which are skewed toward high intrinsic luminosity. Thus the SW Sex phenomenon is probably very common among the cataclysmics with the highest mass transfer rate.' [39] Large mass accretion rates lead to very hot white dwarfs which have been observed e.g. by Gänsicke et al., [9], Hoard et al. [13], and Araujo-Betancor et al. [1]). In fact, as Townsley \& Gänsicke point out, no traditional model of angular momentum loss due to magnetic braking can account for mass accretion rates high enough to explain the observed white dwarf temperatures [41].

Another puzzling feature that has been found in several SW Sex stars is emission-line flaring. Rodríguez-Gil \& Martínez-Pais [28] show that the flares in the SW Sex star V533 Her are periodic (see Figure 6). They thus interpret them as coming from a spinning white dwarf and take it as a
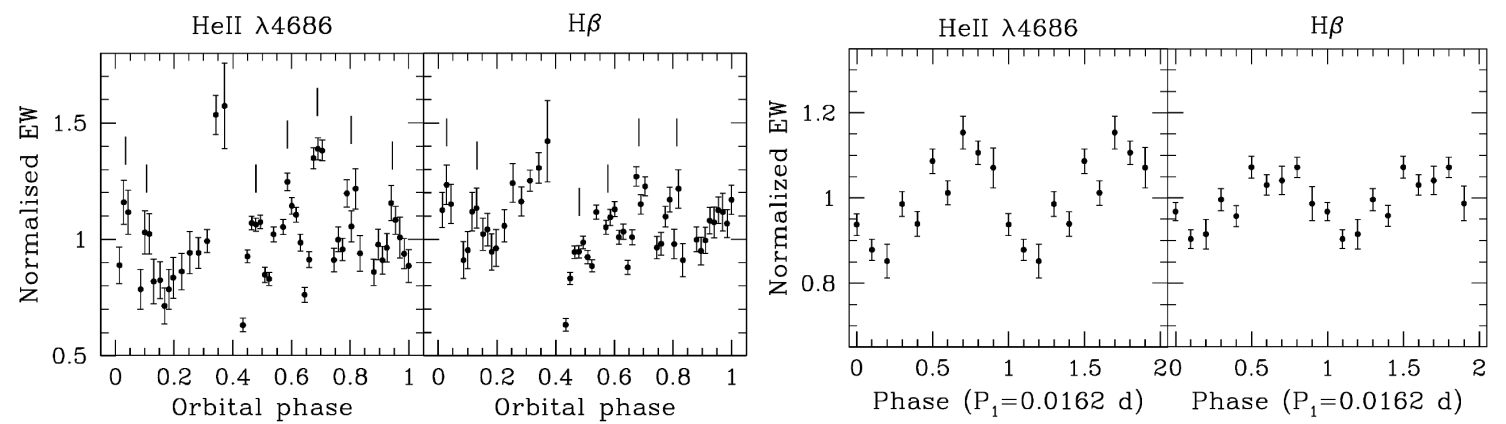

Figure 6: On the left side, the normalised equivalent widths of He II $\lambda 4686$ and $\mathrm{H} \beta$ are plotted against the orbital phase. The flares are indicated with a vertical mark. On the right side, the same data are folded on the 23.33-min period to emphasis the periodicity of the flaring. All plots taken from [28]. 

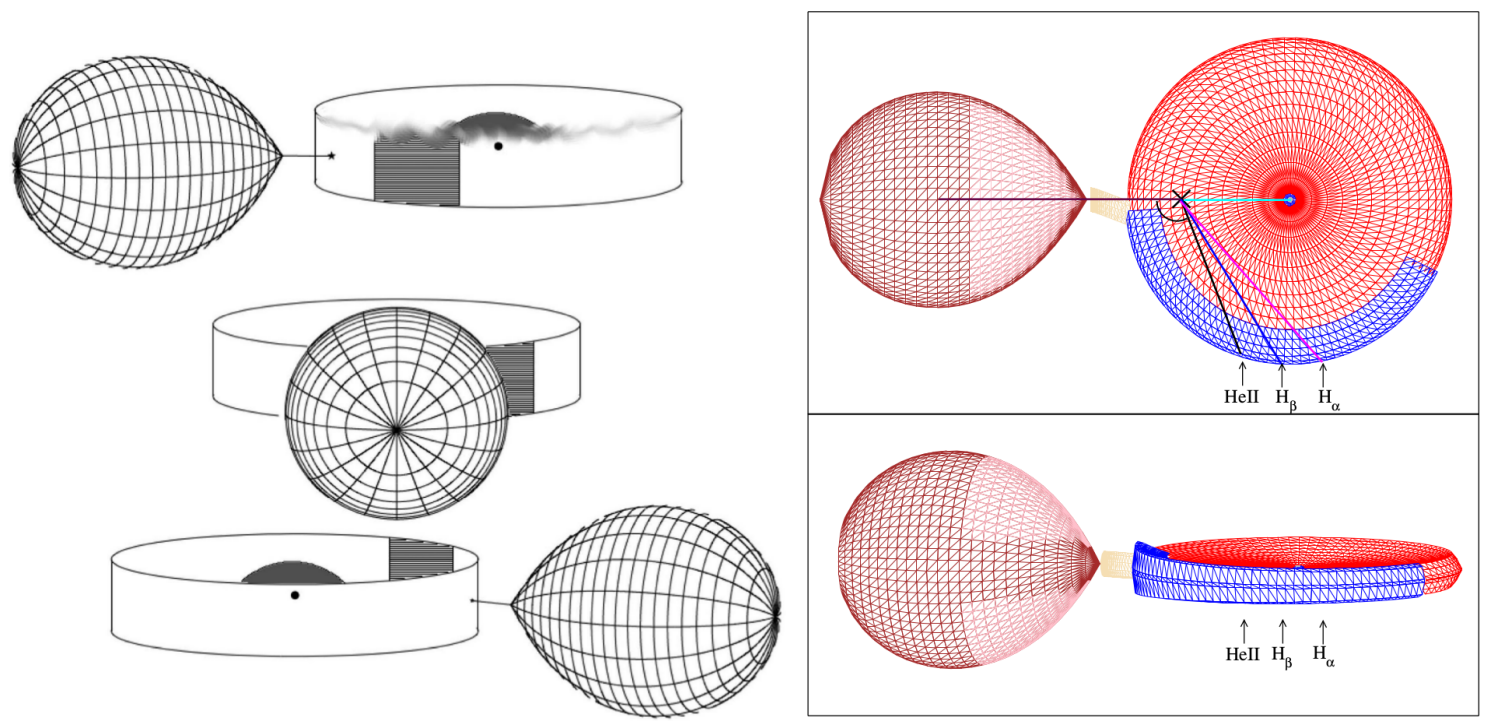

Figure 7: On the right side, a schematic model for DW UMa has been derived by Dhillon et al. [8]. The white dwarf is hidden by the thick accretion disc. Key components of this model are the dominant Balmer emission region downstream of the bright spot on the disc rim (shaded box), the He II emission region close to the white dwarf (shaded arc) and the non-uniform disc edge. Tovmassian et al. [40] developed the geometric model on the right side: The SW Sex system consists of an optically thick (and thus invisible) disc and an extended hot spot region (blue) which is responsible for the single-peaked emission lines. Because of a temperature gradient in the arc, the lines of different elements are emitted in regions that revolve around the centre of mass with different velocity and phase.

evidence for the magnetic nature of the CV. More evidence for the presence of significant magnetism in some SW Sex stars comes e.g. from the detection of variable circular polarisation [29] and variable X-ray emission [3] in LS Peg. Hoard et al. [14] discuss several physical models for SW Sex stars, including simple and complex disc overflow, but also a stream-fed intermediate polar scenario with a truncated inner disc. In this picture, SW Sex stars would be the intermediate polars with the highest mass transfer rates and/or the weakest magnetic fields.

More recent attempts to explain the SW Sex properties use simpler models and forgo the necessity for an out-of-plane component. Assuming an optically thick disc as is observed also in UX UMa stars, Dhillon et al. [8] and Tovmassian et al. [40] explain the single-peaked emission lines as they origin not from the disc (which is optically thick and thus contributes to the continuum but is almost invisible in emission lines) but from an elongated bright spot region where the stream impacts the disc. The presence of an optically thick disc has been confirmed by Knigge et al. [23] from low-state observations of DW UMa where the white dwarf becomes visible and changes the slope of the spectral energy distribution in the UV. More detail on these observations are given in section 3. It remains to mention that the scenario of the emission coming from the bright spot also explains the phase delay observed in all SW Sex stars. By making the edge of the optically thick disc non-uniform, Dhillon et al. could also explain the emission-line flaring in DW UMa [8]. Since they find no periodicity in the line flaring of this system, they rule out a magnetic origin of the flaring in this case. 


\section{SW Sex stars in low state}

Several SW Sex stars are occasionally found in states of greatly diminished brightness, socalled low states or VY-Scl states. During these quiescent phases, the stars are on average 3-5 mag fainter, and they can stay at this level for days, months or even years before returning to the normal high state level. The VY-Scl behaviour is not exclusive to SW Sex stars and also seems to be independent of the magnetic field of the white dwarf. It is observed in almost all polars, some intermediate polars, and many weakly-magnetic CVs like novalikes, dwarf novae, and Z Cam stars. For reviews on such low states in CVs, see [22], [42], and [12].

It is widely accepted, that during such low states, the mass transfer from the donor star through the L1 point is reduced or even completely suppressed, even though the exact mechanism for this cessation of mass supply from the secondary remains unclear. Livio \& Pringle [25] discuss that Roche-lobe overflow can be inhibited by the accumulation of starspots on L1 and this idea is also supported by King \& Cannizzo [22]. Using Roche-Tomography techniques, Watson et al. [43] [44] demonstrated the presence of starspots on the donor stars of CVs and showed that they cluster on the side facing the white dwarf. Hoard et al. [13]) observed MV Lyr during low state and found evidence for the possible presence of starspots on the secondary star near the L1 point. A different approach to explain the phases of subdued mass transfer is taken by Wu et al. [45] who discuss the unstable mass transfer to be caused by an interplay of irradiation, heating and disc shielding of the donor star.

Whatever the reason, mass transfer ceases and the accretion disc decreases in size or disappears completely and the overall system brightness decreases. The main advantage of observing CVs in low states is this weakness or absence of the accretion disc which, especially for SW Sex stars, is
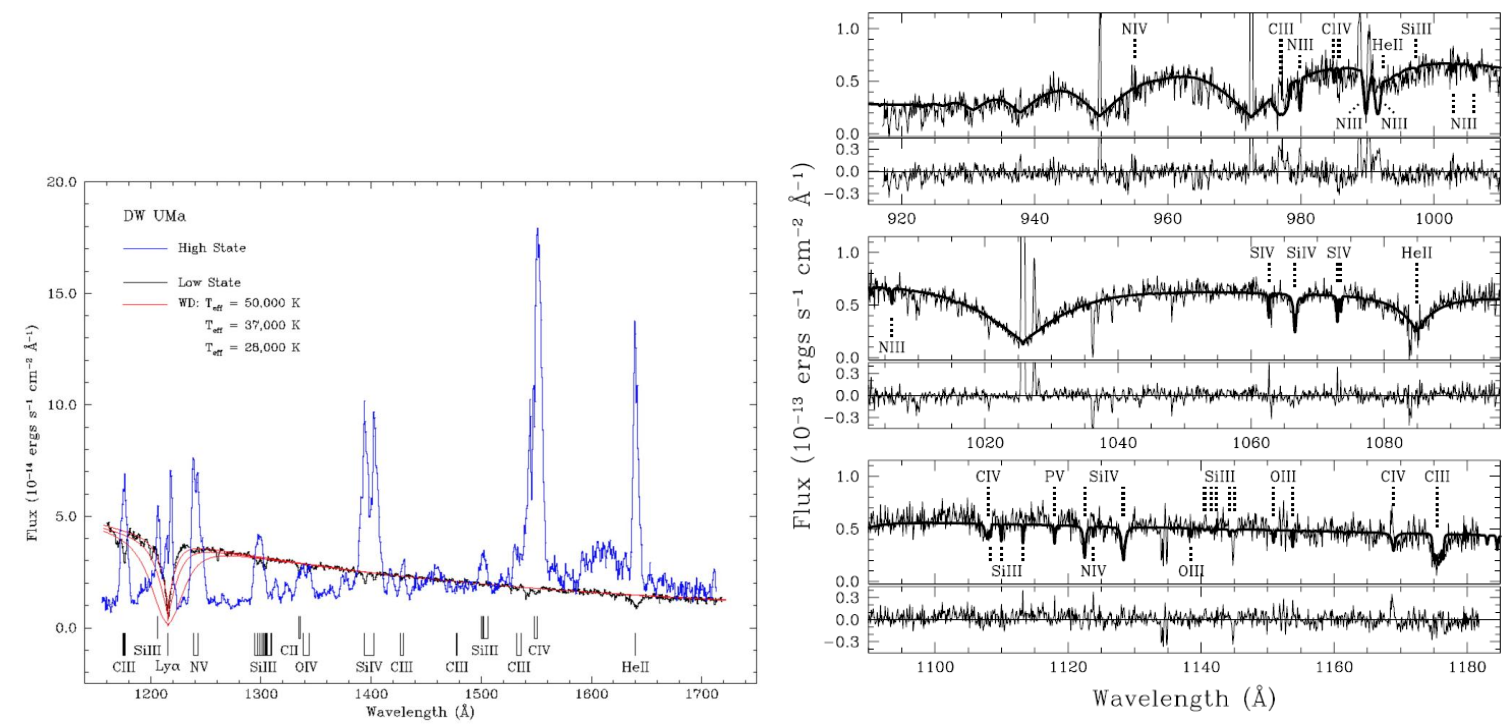

Figure 8: Two examples show how the features of the white dwarf become visible during low state: On the left side, out-of-eclipse UV spectra in the high and low state are plotted for DW UMa. They demonstrate the increased blue flux in low state coming from the white dwarf which in high state is absorbed by the accretion disc [23]) On the right side, the far UV spectrum of MV Lyr is plotted; broad absoption loines from the white dwarf and some narrow emission lines are visible [13]. 
normally the dominating light source in the binary and which is then veiling the emission from the stellar components. Thus, low states provide a unique opportunity to study the white dwarf or the donor star in these systems, to determine system parameters such as temperature, mass, radius, and stellar types from the spectrum and - by following their radial velocity curves over time - to derive the dynamical masses of these components.

Araujo-Betancor et al. [2] analysed ultraviolet (UV) eclipse data of DW UMa that were obtained during a low state in which the UV light was dominated by the hot white dwarf primary. From these, they put new constraints on the binary parameters and the stellar components. Knigge et al. [23] confirm that the UV flux in the low state is higher and bluer than in the high state. This is explained by assuming that in high state, an optically thick accretion disc absorbs the UV emission of the white dwarf (see Figure 8, left side).

Hoard et al. [13]) obtained a far-ultraviolet spectrum (see Figure 8, right side), time-resolved optical photometry, and optical spectroscopy of MV Lyr in low state. By combining these data, they establish a model for the white dwarf, the secondary star and the binary system. They find no evidence for the presence of an accretion disc.

In $2008, \mathrm{BB}$ Doradus faded from $\mathrm{V} \approx 14.3$ towards a deep low state at $\mathrm{V} \approx 19.3$. During this low state, it was monitored photometrically and spectroscopically by Rodríguez-Gil et al. [31] and Schmidtobreick et al. [34]. They observed episodic accretion events which veiled the absorption spectra of the white dwarf and the donor and radically changed the line profiles on timescale of
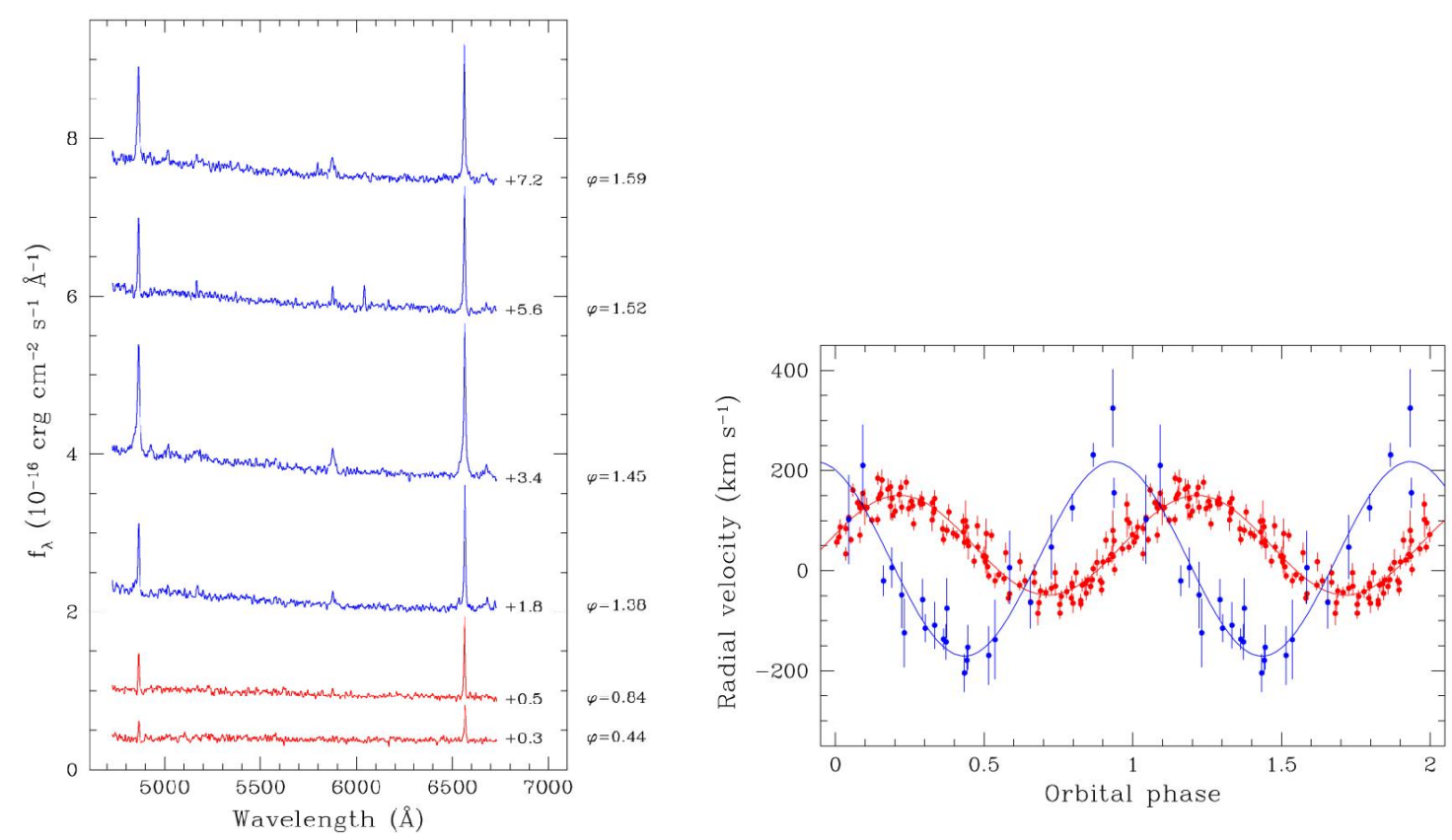

Figure 9: The sporadic mass transfer events in the low state of BB Dor are demonstrated: On the left, an observing sequence of about four hours of is plotted, on the right the $\mathrm{H} \alpha$ radial velocities in quiescence (red dots) and during the accretion events (blue dots). The solid lines are the respective best sine fits. The phases of the radial velocities from the quiescent spectra indicate that the origin lies in the irradiated donor star. The radial velocities during the accretion events are delayed by 0.18 cycle with respect to the expected motion of the white dwarf and thus do not origin in a disc around the white dwarf but rather in a stream or impact region. [31]. 

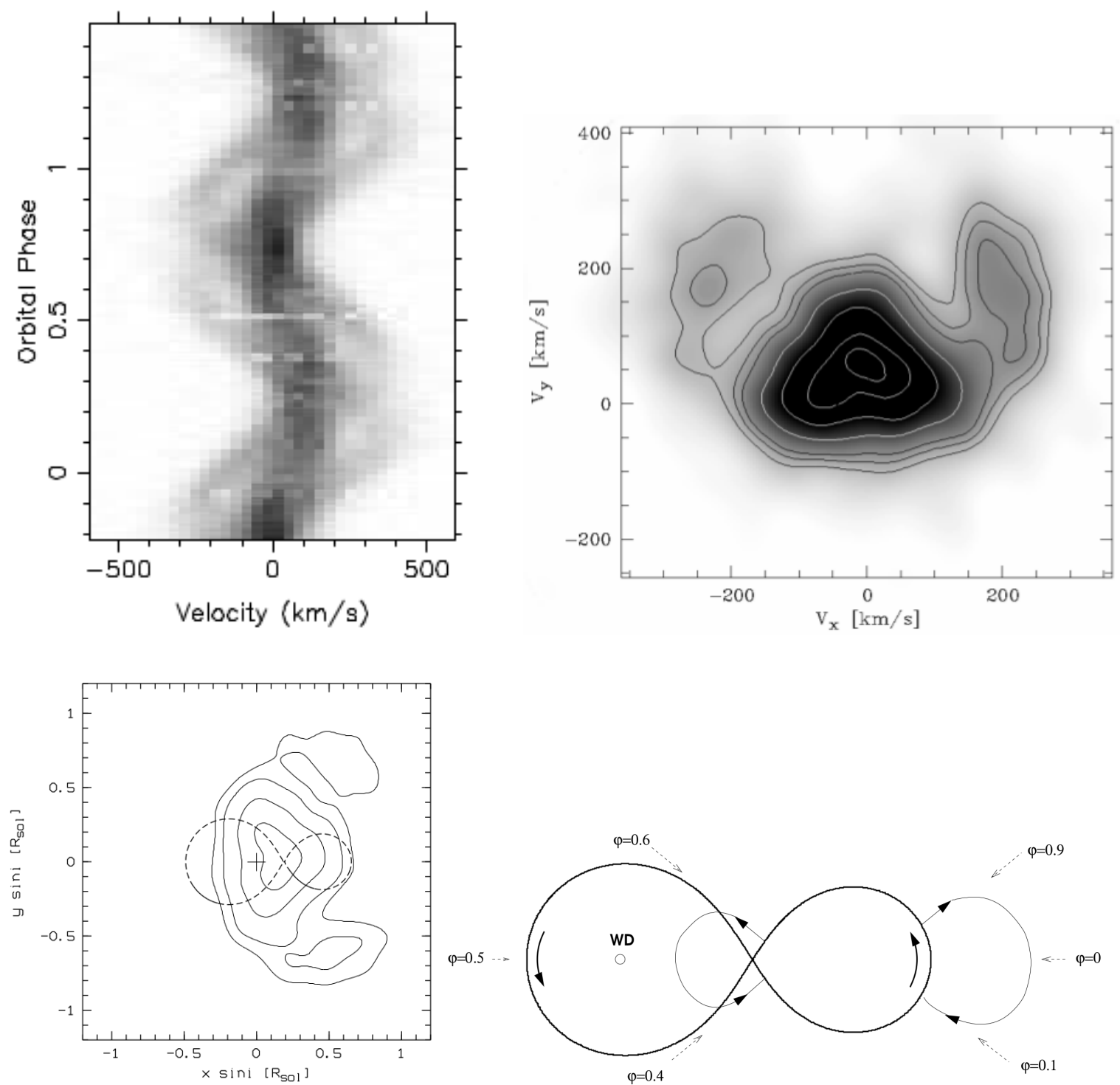

Figure 10: The four plots are taken from Schmidtobreick et al. [34] and show the presence and interpretation of satellite lines in BB Dor in low state. On the upper left the trailed spectrograms of the $\mathrm{H} \alpha$ line are plotted. The strong line moving in the centre is attributed to the irradiated secondary star. The satellite lines following two crossing sinusoidal curves are clearly visible. The upper right plot shows the Doppler map of the $\mathrm{H} \alpha$ emission sources in the system. Assuming that all emission sources are bound to the orbit, the velocities of the $\mathrm{H} \alpha$ emission can be converted into Cartesian coordinates (see lower left plot). The sketch on the lower right side illustrates the two possible orientations of prominences that could explain the observed satellite lines. Scaling and sizes are arbitrary.

tens of minutes: the narrow emission lines which originate in the irradiated secondary switched to broader lines with strong wings, and high-excitation lines such as He II and the Bowen blend appeared. A shift of about 0.35 phases between the radial velocities of the narrow lines and those of the broad line wings is found. This shows that accretion is not complete quenched in the low state but can be switched on fast, possibly triggered by flaring activity on the secondary star [31]. Schmidtobreick et al. [34] analysed quiescence data taken outside such accretion events. The spec- 

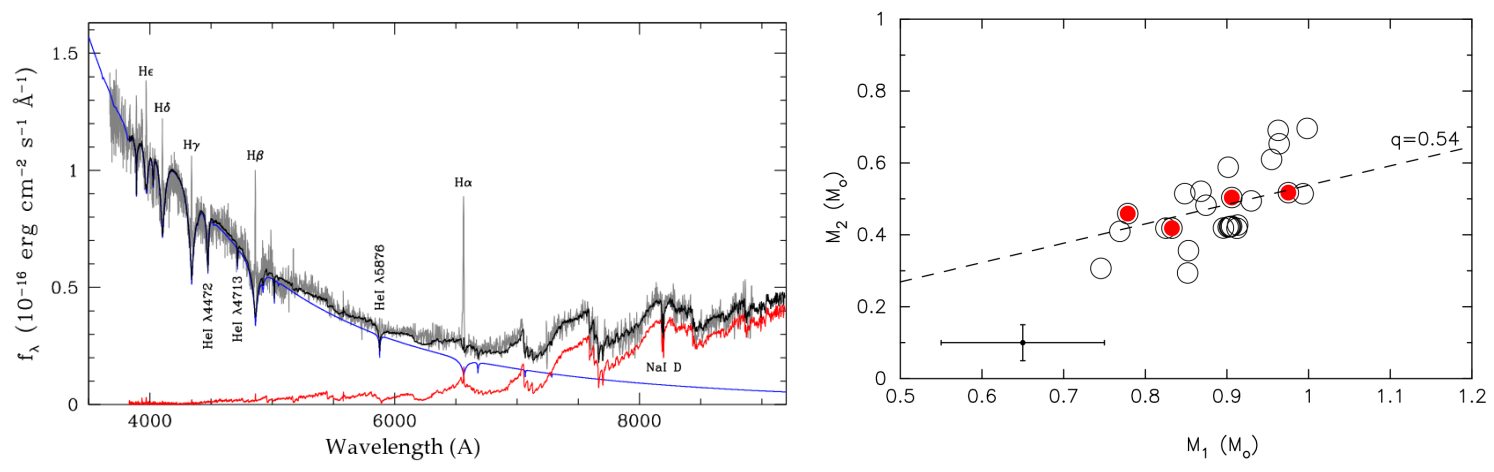

Figure 11: The left presents the average quiescence spectrum of HS 0220+0603. The data (grey) are fitted with white dwarf (blue) + M-dwarf (red) templates. On the right, the $M_{1}-M_{2}$ plane is plotted with the best-fitting solutions ( 99 per cent confidence level for red circles) The dashed line corresponds to a mass ratio of $\mathrm{q}=0.54$. [30]

tra show narrow emission lines of $\mathrm{H} \alpha$, $\mathrm{He}$ I and $\mathrm{Na}-\mathrm{D}$, as well as $\mathrm{TiO}$ absorption troughs which trace the motion of different temperature zones on the irradiated secondary star. While $\mathrm{He}$ I and $\mathrm{H} \alpha$ originate at the higher temperature regions of the secondary star close to L1 (which is reflected in their low radial-velocity amplitude), the TiO bands are likely to originate in the non-irradiated, cooler, backside zones of the secondary star (high radial-velocity amplitude). No signature of accretion is present in the spectra. However, they detect two additional components in the $\mathrm{H} \alpha$ emission line, satellite lines similar to what has been detected previously in AMHer stars [17] [18] [19] [20] [21] [26]. A modified Roche geometry taking into account an additional magnetic force might result in stable equilibrium points around the positions where the increased emission is observed. A different possibility is that the $\mathrm{H} \alpha$ emitting material moves along prominences originating in a magnetically active secondary star. To explain the observed variation of the satellite lines, only two orientations are possible for these prominences (see Figure 10 for the data and a schematic interpretation)

The last example of an analysis of low-state data of a SW Sex system refers to the eclipsing star HS 0220+0603 which was extensively observed during the 2004-2005 low state by Rodríguez-Gil et al. (2015) [30]. Their dataset include time-resolved spectroscopy and photometry in various optical bands. Both the white dwarf and the secondary star are visible in the optical spectra. Spectral modelling revealed the secondary to be of type M5.5 with an effective temperature of $2800 \mathrm{~K}$. The white dwarf turned out to be of DAB type and with an effective temperature of $30.000 \mathrm{~K}$. Simultaneously modelling of the eclipse lightcurve and the radial velocities of both components, allowed to derive dynamical stellar masses for the first time (Figure 11).

\section{SW Sex stars as an evolutionary state}

In general, the evolution of stable contact binaries is driven by the angular momentum loss which as such determines the mass transfer rate. This implies that the general evolutionary direction for CVs is from longer to shorter orbital periods. According to the standard model of CV evolution, the main source of angular momentum loss for CVs with orbital periods $P_{\text {orb }}>3 \mathrm{~h}$ is the magnetic braking. Due to the continuous mass transfer, the secondary is pushed out of thermal 
equilibrium and becomes bloated. At a period of about $3 \mathrm{~h}$, i.e. at the upper edge of the period gap, the secondary becomes fully convective, magnetic braking ceases and only the much weaker braking via gravitational radiation continues. This allows the star to approach thermal equilibrium and to shrink to the volume corresponding to its mass. It thus loses contact with its Roche lobe, mass transfer stops and the $\mathrm{CV}$ enters the period gap. For an extensive review of the current understanding of CV evolution, see Knigge et al. [24].

Given their position in the period distribution, CVs with orbital periods between $3 \mathrm{~h}$ and $4 \mathrm{~h}$ thus play a major role in our understanding of CV evolution. They are the ones at the upper edge of the period gap whose donor stars are predicted to become fully convective and where magnetic braking is about to cease so they will become a detached CV in the next future. Rodríguez-Gil et al. [32] have shown that at least 50\% of all CVs in this period regime are SW Sex stars, novalikes with extremely high mass accretion rates leading to very hot white dwarfs. As mentioned earlier, no angular momentum loss by traditional magnetic braking can account for such high mass transfer rates and white-dwarf temperatures [41]. So the question arises whether this unusual high mass transfer rate that manifests itself just for these binaries where major changes in the donor structure are supposed to happen, is in any way connected to these changes. As far as I am aware, only one approach has been made to explain the high mass transfer rate above the period gap in terms of CV evolution. Zangrilli et al. [46] assume that for the CVs above the period gap, only the convective envelope of the donor is co-rotating with the binary while the radiative core remains at a lower rotation velocity. They calculate the angular momentum loss arising from a combination of two $\alpha-\Omega$ dynamos, one in the envelope and one at the boundary between core and envelope. By the time the radiative core disappears, the boundary dynamo becomes the dominant source of magnetic braking and would the mass transfer rates for those CVs that inhabit the upper edge of the period gap, the SW Sex stars.

Schmidtobreick et al. [35] demonstrate that all the novalike-stars in their sample of nonmagnetic CVs with periods between 3 and 4 hours that have sufficient data observed, show at least some SW Sex characteristics. They argue that the nova-like population in the $3-4 \mathrm{~h}$ period regime is clearly dominated by SW Sex stars and almost all CVs in this period regime show the extremely high mass transfer rates. Since according to the standard model, all long period CVs have to evolve through this period regime before entering the period gap, they should then share the SW Sex characteristics during that time. In fact, the orbital period distribution shows a decrease of dwarf novae around $4 \mathrm{~h}$ just where the SW Sex stars begin to appear (see Figure 12). One can assume that their mass transfer ceases earlier than for nova-like stars or that they evolve into the high mass-transfer systems. In either case, the dominant SW Sex phenomenon between $3 \mathrm{~h}$ and $4 \mathrm{~h}$ is a phase in the secular evolution of CVs that challenges the standard model of CV evolution.

\section{Summary}

When they were originally discovered, SW Sex stars were considered peculiar systems, characterised by enigmatic properties that disagreed with the general understanding of CVs and their components. During the last decades it became, however, clear that these systems represent a large fraction of CVs and actually dominate the population at the upper edge of the period gap. The SW Sex characteristics can be explained by assuming high mass transfer rates leading to hot accre- 


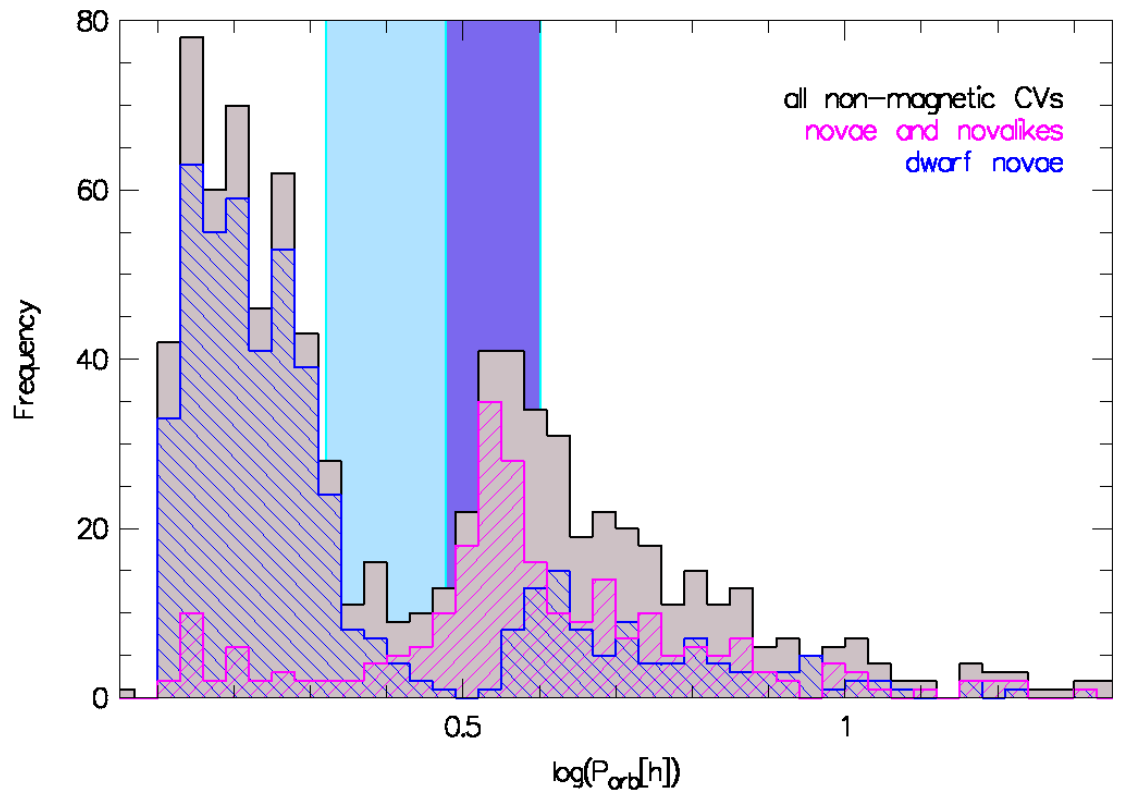

Figure 12: The orbital period distribution for different subtypes of non-magnetic CVs has been built using the data from Ritter \& Kolb (2003), update RKcat7.18, 2012. The position of the period gap is indicated by the light blue bar, the darker bar indicates the 3-4 h range dominated by systems with high mass transfer. The number of dwarf novae decreases at the upper edge of the SW-Sex regime.

tion streams, optically thick discs, and outflows that in combination produce the observed features. The definition of SW Sex stars has become broader, aiming more for the physical difference rather then the phenomenology. Thus, we now consider the SW Sex stars as those nova-like CVs with the most extreme mass transfer rates. The reason why they accumulate in the orbital period range above the gap is still unknown, but a connection to the secular evolution of CVs in this range seems likely.

\section{References}

[1] Araujo-Betancor S., Gänsicke B. T., Hagen H.-J., Marsh T. R., Harlaftis E. T., Thorstensen J., Fried R. E., Schmeer P., Engels D., 2005, A\&A 430, 629

[2] Araujo-Betancor S., Knigge C., Long K. S., Hoard D. W., Szkody P., Rodgers B., Krisciunas K., Dhillon V. S., Hynes R. I., Patterson J., Kemp J. 2003, ApJ 583, 437

[3] Baskill D. S., Wheatley P. J., Osborne J. P., 2005, MNRAS 357, 626

[4] Beuermann K., Thorstensen J. R., Schwope A. D., Ringwald F. A., Sahin H. 1992, A\&A 256, 442

[5] Casares J., Martínez-Pais I. G., Marsh T. R., Charles P. A., Lázaro C. 1996, MNRAS 278, 219

[6] Dhillon V. S., 1990, PhD Thesis

[7] Dhillon V. S., Jones D. H. P., Marsh T. R., Smith R. C. 1992, MNRAS 258, 225

[8] Dhillon V. S., Smith D. A., Marsh T. R. 2013, MNRAS 428, 3559

[9] Gänsicke B. T., Sion E. M., Beuermann K., Fabian D., Cheng F. H., Krautter J., 1999, A\&A 347, 178 
[10] Gänsicke B. T. 2005, ASP Conf. Ser. 330, 3

[11] Hellier C. 1996, ApJ 471, 949

[12] Hessman F. V., 2000, NewAR 44, 155

[13] Hoard D. W., Linnell A. P., Szkody P., Fried R. E., Sion E. M., Hubeny I., Wolfe M. A. 2004, ApJ 604,346

[14] Hoard D. W., Szkody P., Froning C. S., Long K. S.Knigge C. 2003, AJ 126, 2473

[15] Honeycutt R. K., Schlegel E. M., Kaitchuck R. H., 1986, ApJ 302, 388

[16] Horne K., Marsh T. R. 1986, MNRAS 218, 761

[17] Kafka S., Honeycutt R. K., Howell S. B., Harrison T. E, 2005, AJ 130, 2852

[18] Kafka S., Honeycutt R. K., Howell S. B., 2006, AJ 131, 2673

[19] Kafka S., Howell S. B., Honeycutt R. K., Robertson J. W., 2007, AJ 133, 1645

[20] Kafka S., Ribeiro T., Baptista, R., Honeycutt R. K., Robertson J. W., 2008, ApJ 688, 1302

[21] Kafka S., Tappert C., Ribeiro T., Honeycutt R. K., Hoard D. W., Saar, S., 2010, ApJ 721, 1714

[22] King A. R., Cannizzo J. K., 1998, ApJ 499, 348

[23] Knigge C., Araujo-Betancor S., Gänsicke B. T., Long K. S., Szkody P., Hoard D. W., Hynes R. I., Dhillon V. S. 2004, ApJ 615, L129

[24] Knigge C., Baraffe I., Patterson J., 2011, ApJS 194, 28

[25] Livio M., Pringle J. E., 1994, ApJ 427, 956

[26] Mason E., Howell S. B., Barman T., Szkody P., Wickramasinghe D., 2008, A\&A 490, 279

[27] Ritter H., Kolb U., 2003, A\&A 404, 301

[28] Rodríguez-Gil P., Martínez-Pais I. G. 2002, MNRAS 337, 209

[29] Rodríguez-Gil P., Casares J., Martínez-Pais I. G., Hakala P., Steeghs D., 2001, ApJ 548, L49

[30] Rodríguez-Gil P., Shahbaz T., Marsh T. R., Gänsicke B. T., Steeghs D., Long K. S., Martínez-Pais I. G., Armas Padilla M., Schwarz R., Schreiber M. R., Torres M. A. P., Koester D., Dhillon V. S., Castellano J., Rodríguez D. 2015, MNRAS 452, 146

[31] Rodríguez-Gil P., Schmidtobreick L., Long K. S., Gänsicke B. T., Torres M. A. P., Rubio-Díez M. M., Santander-García M. 2012, MNRAS 422, 2332

[32] Rodríguez-Gil P., Gänsicke B. T., Hagen H.-J., Araujo-Betancor S., Aungwerojwit A., Allende Prieto C., Boyd D., Casares J., Engels D., Giannakis O., Harlaftis E. T., Kube J., Lehto H., Martínez-Pais I. G., Schwarz R., Skidmore W., Staude A., Torres M. A. P. 2007(A), MNRAS 377, 1747

[33] Rodríguez-Gil P., Schmidtobreick L., Gänsicke B. T. 2007(B), MNRAS 374, 1359

[34] Schmidtobreick L., Rodríguez-Gil P., Long K. S., Gänsicke B. T., Tappert C., Torres M. A. P. 2012(A), MNRAS 422, 731

[35] Schmidtobreick L., Rodríguez-Gil P., Gänsicke B. T. 2012(B), Mem. Societa Astronomica Italiana 83, 610

[36] Schmidtobreick L., T., Tappert C., Saviane I. 2003 MNRAS 342, 145

[37] Smith D. A., Dhillon V. S., Marsh T. R. 1998, MNRAS 296, 465 
[38] Thorstensen J. R., Taylor C.J. 2000, MNRAS 312, 629

[39] Thorstensen J. R., Ringwald F. A., Wade R. A., Schmidt G. D., Norsworthy J. E., 1991, AJ 102, 272

[40] Tovmassian G., Stephania Hernandez M., González-Buitrago D., Zharikov S., García-Díaz M. T., 2014, AJ 147, 68

[41] Townsley D. M. and Gänsicke B. T., 2009, ApJ 693, 1007

[42] Warner B., 1999, ASP Conf. Ser. 157, 63

[43] Watson C. A., Dhillon V. S., Shahbaz T., 2006, MNRAS 368, 637

[44] Watson C. A., Steeghs D., Dhillon V. S., Shahbaz T., 2007, AN 328, 813

[45] Wu K., Wickramasinghe D. T., Warner B., 1995, PASA 12, 60

[46] Zangrilli L., Tout C. A., Bianchini A., 1997, MNRAS 289, 59 